\title{
Pseudoaneurysmal Formation in Abdominal Aorta Associated with Escherichia coli Infection
}

\author{
Takuya Furuta ${ }^{1}$, Toshinari Tsubokawa ${ }^{1}$, Shu Takabatake ${ }^{1}$, Hiroshi Ohtake ${ }^{2}$, \\ Go Watanabe ${ }^{2}$ and Masakazu Yamagishi ${ }^{1}$
}

\begin{abstract}
Early and accurate diagnosis of infective aortic aneurysms (IAA) is critical for adequate treatment to optimize patient outcome. We report the case of an 84-year-old man who complained of severe back pain with high fever and was finally diagnosed as Escherichia coli-related IAA. Computed tomography showed a periaortic soft tissue density and irregular fringe adjacent to the non-dilated abdominal aorta suggesting the presence of pseudoaneurysm. In addition to intravenous antibiotic injection, an aneurysmectomy with extensive debridement and an in situ graft, were successfully performed. The case emphasizes the potential for rapid IAA change and the need for frequent radiologic follow-up.
\end{abstract}

Key words: infective aortic aneurysm, E. coli, contrast-enhanced CT

(Intern Med 50: 1025-1028, 2011)

(DOI: 10.2169/internalmedicine.50.4370)

\section{Introduction}

Infective aortic aneurysms with Escherichia coli are rare and difficult to treat. Few data exist regarding the long-term prognosis and survival rate due to early diagnosis and prompt surgical and antibiotic therapy of IAA. This report describes a case in which contrast-enhanced computed tomography was useful for detection of disease progression, and the problems associated with management are also discussed.

\section{Case Report}

An 84-year-old man was admitted to the local hospital because of fever, general malaise and lower back pain. The patient had a 12-year history of type 2 diabetes mellitus, hypertension and prostatic hyperplasia. The diabetes therapy with insulin was poorly control (hemoglobin A1c 8.6\%) and antihypertensive medication was also insufficient (blood pressure $150-165 / 85-95 \mathrm{mmHg}$ ) by the outpatient care. Laboratory tests revealed leukocytosis $\left(15,800 / \mathrm{mm}^{3}\right)$ with neutrophilia (Fig. 1) and elevation of serum levels of C- reactive protein $(14.6 \mathrm{mg} / \mathrm{dL})$. There was no evidence for urinary tract infection. A chest X-ray did not show an infiltrative lesion. The first abdominal computed tomography (CT) on admission to the local hospital showed a periaortic soft tissue mass and fringe irregular adjacent to a nondilated abdominal aorta near the inferior mesenteric artery with air pocket. There also existed a thin hypodense rim between this density and the aortic mural calcification (Fig. 2). These findings suggested the differential diagnosis including IAA, infectious or neoplastic lymphadenopathy, and retroperitoneal abscess. Escherichia coli (E-coli) was isolated from blood culture. Thus, the patient was diagnosed as E. colirelated IAA. Intravenous antibiotic treatment was commenced as bacteremia using cefepim (4 g/day) and clindamycin (1.2 g/day) to cover isolated bacilli and anaerobe.

Although the laboratory data temporarily improved after starting antibiotics, he was transferred to our hospital on the 5th hospital day due to the high risk of aortic rupture. On admission to our hospital, the patient complained of mild tenderness in the mid abdomen and mild leg edema. On physical examination, his blood pressure was $154 / 85 \mathrm{mmHg}$, pulse rate was $83 / \mathrm{min}$, and body temperature was $36.4^{\circ} \mathrm{C}$. On the 5th day after admission (10th hospital day), the pa-

${ }^{1}$ Division of Cardiovascular Medicine, Kanazawa University Graduate School of Medicine, Japan and ${ }^{2}$ Department of General and Cardiothoracic Surgery, Kanazawa University Graduate School of Medicine, Japan

Received for publication August 15, 2010; Accepted for publication January 17, 2011

Correspondence to Dr. Toshinari Tsubokawa, toshi_s1209@yahoo.co.jp 


\section{Clinical course}

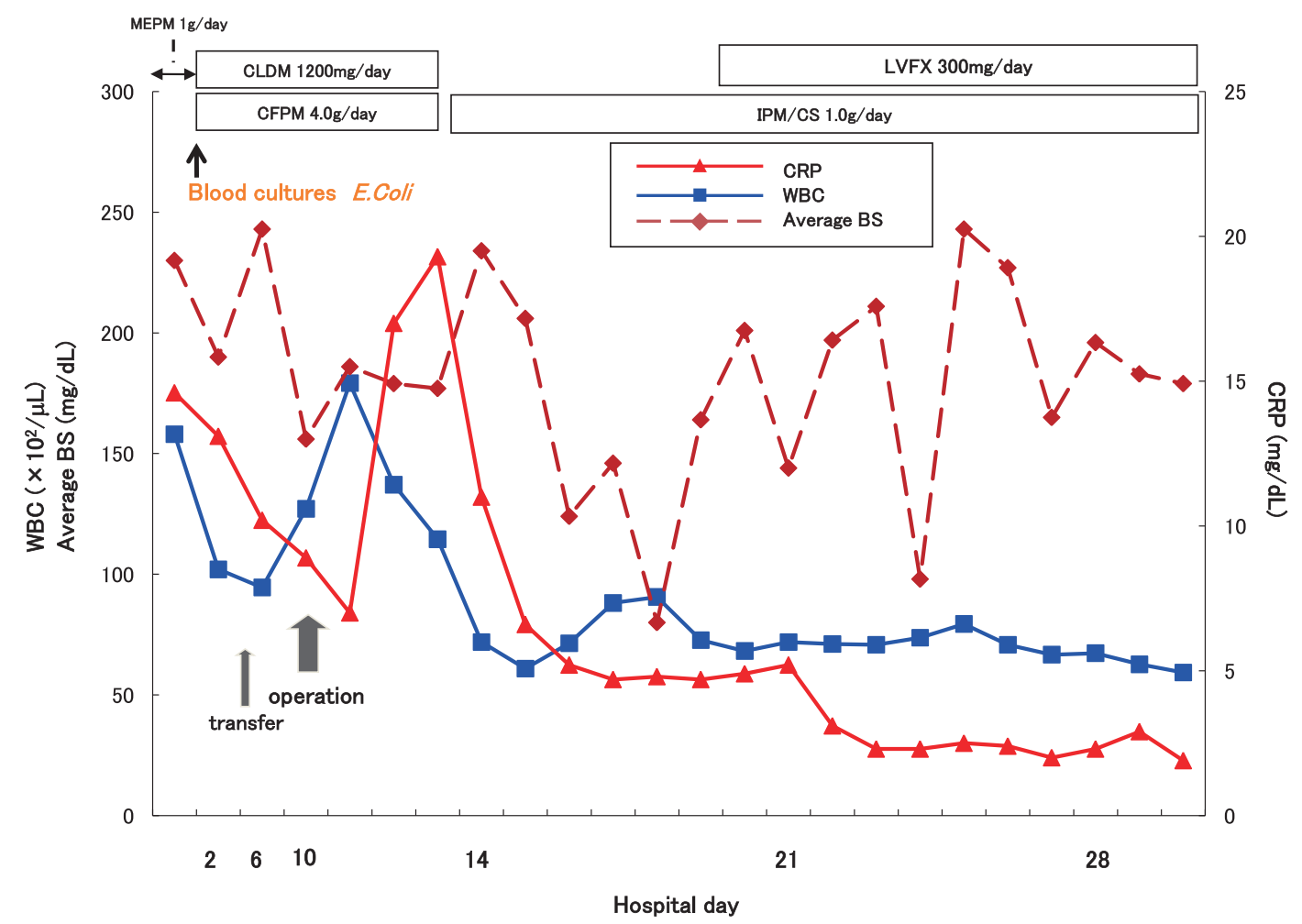

Figure 1. Patient's clinical course. Changes in the count of white blood cells (WBC) per microliter of blue blood, the serum level of C-reactive protein (CRP: red blood) and average blood sugar (BS: brown) are shown. MEPM: meropenem, CLDM: clindamycin, CFPM: cefepime, LVFX: levofloxacin, IPM/CS: imipenem/cilastatin

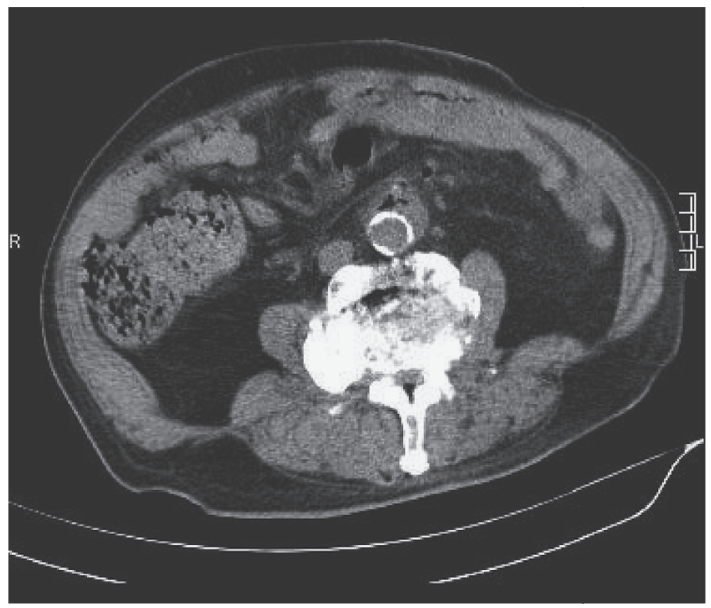

(A)

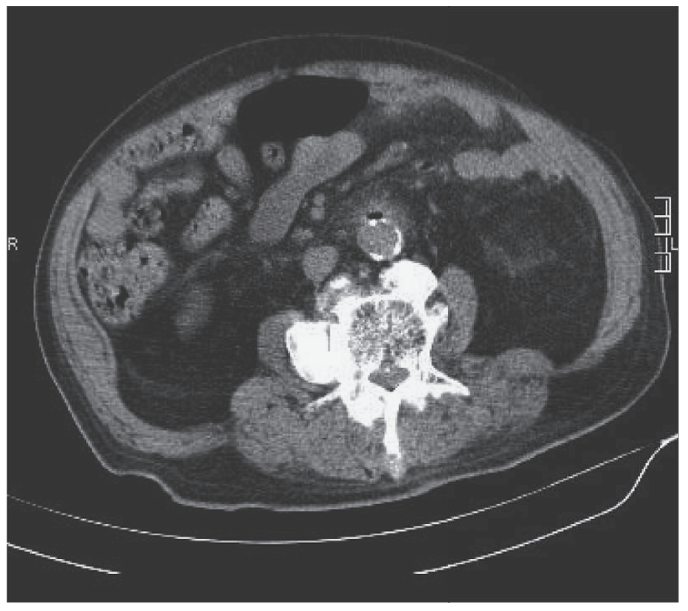

(B)

Figure 2. Computed tomography (CT) images at the local hospital. Abdominal computed tomography showed periaortic soft tissue mass with air pocket (A). There are calcified plaques in the aorta with irregular fringe $(B)$.

tient's lower back pain gradually worsened without significant physical findings. His white blood cell count was elevated to $12,700 / \mathrm{mm}^{3}$ (Fig. 1), and hemoglobin value ( $\mathrm{Hb} 8.4$ $\mathrm{g} / \mathrm{dL}$ ) was decreased. Although he has moderate renal dysfunction (Cr 1.1-1.4 mg/dL, GFR 35-46 mL/min), there was no aggravation in the hospital. An abdominal X-ray showed deformity of the vertebral body and normal colon gas, and did not show non-specific findings such as disappearance of the psoas line and displacement of calcific aorta (Fig. 3). On the same day, contrast-enhanced CT scan with hydration demonstrated a saccular pseudo-aneurysm with an irregular lumen and inhomogeneous thick wall, and extending from 
the infrarenal region to the inferior mesenteric artery (Fig. 4); this suggested the possible occurrence of IAA rupture.

The patient underwent emergency open repair of the large pseudo-aneurysm. At surgery, there was a large volume of pus in the aneurysm. After the aneurysm and surrounding

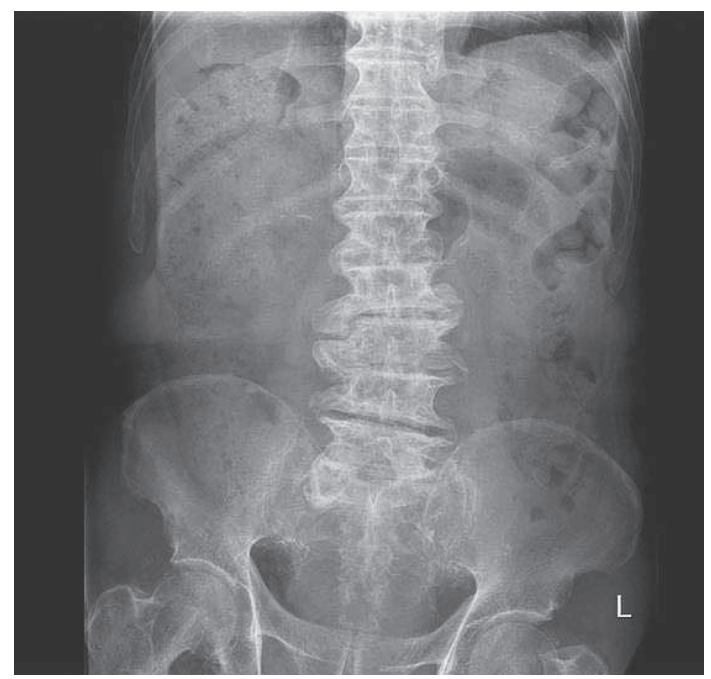

Figure 3. An abdominal X-ray did not show significant findings.

(A)

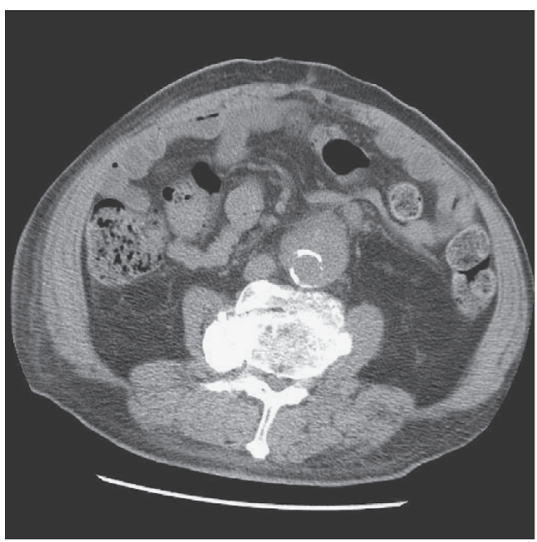

(B)

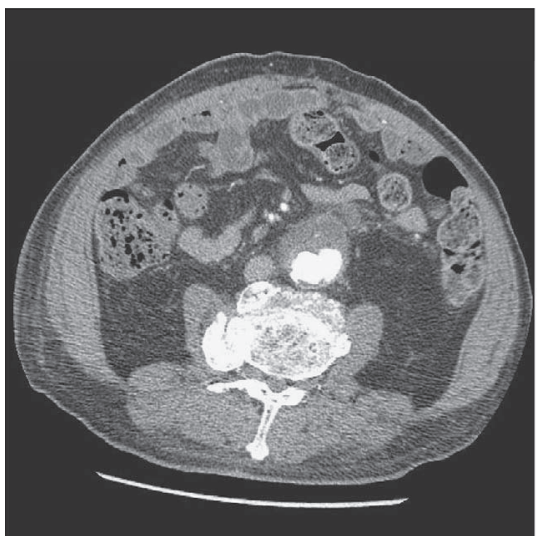

inflammatory tissues were completely removed, a descending aortic replacement with a Hemashield platinum graft $(14 \times 7 \mathrm{~mm})$ was performed. The culture of the resected tissues and drainage fluid grew $E$. coli sensitive to the antibiotics already used for the patient. After the surgery, the CTscan showed good position of prosthetic graft and no evidence of infection. The patient's symptoms were resolved, and there were no complications. He was discharged well on post-operative day 28 and received antibiotic administration with levofloxacin for 6 months.

\section{Discussion}

The prevalence of IAA in the abdominal aorta is $0.7-1.3 \%$ of all surgically treated aortic aneurysms (1-5). The mortality of IAA is high and depends upon the artery involved, treatments received, and underlying conditions. Some reports of IAA demonstrated a perioperative mortality of 21$36 \%(3,6)$. Seven to $24 \%$ of IAA demonstrated free rupture and a further $47-61 \%$ demonstrate contained or impeding rupture at presentation and freely ruptured infected aneurysms have $63-100 \%$ mortality $(3,6)$. A number of routes account for infection of an arterial wall including septic emboli, bacteremic seeding of an existing intimal injury or atherosclerotic plaque, contiguous involvement of the vessel

(C)

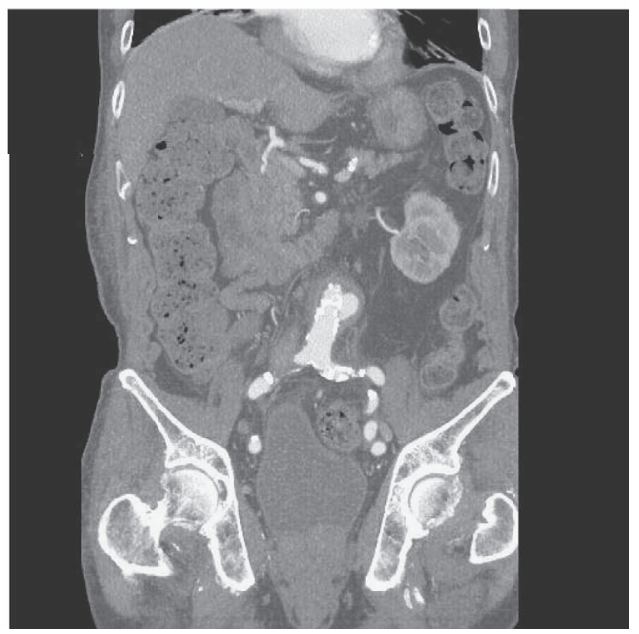

Figure 4. Aneurysmal change of the infected aorta. Abdominal CT on the day the symptoms emerged. By plain CT, the periaortic soft tissue mass was enlarged and the periaortic gas vanished (A). By contrast-enhanced CT (axial), a large saccular aneurysm was extended and still surrounded by soft tissue mass. Note that the left lateral aortic wall was disrupted (B). Coronal reconstruction at the same time showed a saccular shape of the pseudo-aneurysm (C). 
from an adjacent source of sepsis, and direct bacterial inoculation at the time of trauma. Gram-negative bacteria such as E. coli and Klebsiella used to be considered as a less common cause of this situation. These bacteria have become more frequent to be $7-14 \%$ of IAA $(3,6)$ which was preceded by urinary tract infection (7).

The present case had a history of prostatic hyperplasia sometimes associated with prostatitis. It suggests the possibility of bacteremic seeding of an exisiting intima injury, although there was no evidence for $E$. coli infection of the prostate.

Together with contrast-enhanced CT scan signs such as a leakage of aortic contrast material, air in the aortic wall, a rapid growing or newly developed aneurysm, a saccular aneurysm, or a soft tissue mass surrounding the aneurysm aorta, are suggestive of IAA, and early operative intervention is indicated (8-10). Because of the acute IAA expansion, elevated inflammatory response and aggravation of symptoms, we suspected IAA with possible impending rupture. We therefore decided to perform emergency surgery. The cause leading to IAA rupture may be high blood pressure, easily infective and arterial intimal embrittlement with DM.

Early detection of IAA is critically important for adequate treatment to optimize patient outcome. However, it is somewhat difficult to determine IAA until impending rupture, because this can rapidly develop or enlarge. Although a low invasive test, such as abdominal X-ray or echography, is beneficial for patients with renal dysfunction and allergy of contrast agent, and it is useful for a diagnosis of aneurysmal size and localization of the lesion, it is difficult to assess extent of infection and aneurysmal rupture. Magnetic resonance angiography can also define disease extension and facilitate planning of surgical intervention (11) as the alternative diagnostic method. But, it is important to confirm the detection of signs of rupture by proving on imaging and by worsening of clinical data.

In summary, we presented a case with IAA caused by E- coli infection. The present report emphasizes the importance of sequential contrast-enhanced CT examinations for suspected cases, because infective aneurysms may lead to rupture with high mortality.

The authors state that they have no Conflict of Interest (COI).

\section{References}

1. Klontz KC. Frequency of infected aneurysms among patients in Department of Veterans Affairs Hospitals, 1986-1990: the role of Salmonella. Mil Med 162: 766-768, 1997.

2. Chan FY, Crawford ES, Coselli JS, Safi HJ, Williams TW Jr. In situ prosthetic graft replacement for mycotic aneurysm of the aorta. Ann Thorac Surg 47: 193-203, 1989.

3. Muller BT, Wegener OR, Grabitz K, Pillny M, Thomas L, Sandmann W. Mycotic aneurysms of the thoracic and abdominal aorta and iliac arteries: experience with anatomic and extra- anatomic repair in 33 cases. J Vasc Surg 33: 106-113, 2001.

4. Brown SL, Busuttil RW, Baker JD, Machleder HI, Moore WS, Barker WF. Bacteriologic and surgical determinants of survival in patients with mycotic aneurysms. J Vasc Surg 1: 541-547, 1984.

5. Hsu RB, Chen RJ, Wang SS, Chu SH. Infected aortic aneurysms: Clinical outcome and risk factor analysis. J Vasc Surg 40: 30-35, 2004.

6. Oderich GS, Panneton JM, Bower TC, et al. Infected aortic aneurysms: aggressive presentation, complicated early outcome, but durable results. J Vasc Surg 34: 900-908, 2001.

7. Khaira HS, Allan A, Crowson MC. Escherichia coli infection of an abdominal aortic aneurysm. J Infect 32: 161-163, 1996.

8. Lee WK, Mossop PJ, Little AF, et al. Infected (mycotic) aneurysms: spectrum of imaging appearances and management. Radiographics 28: 1853-1868, 2008.

9. Rozenblit A, Bennett J, Suggs W. Evolution of the infected abdominal aortic aneurysm: CT observation of early aortitis. Abdom Imaging 21: 512-514, 1996.

10. Phade SV, deFreitas D, Powell CS, Stoner M. Evolution of bacterial arteritis into a mycotic aortic aneurysm. Vasc Endovascular Surg 41: 158-160, 2007.

11. Corso JE, Kasirajan K, Milner R. Endovascular management of ruptured, mycotic abdominal aortic aneurysm. Am Surg 71: 515517, 2005.

(C) 2011 The Japanese Society of Internal Medicine http://www.naika.or.jp/imindex.html 